\title{
Smoking cessation in pregnancy
}

\author{
Renee Bittoun and Giuseppe Femia BSc MBBS
}

Brain and Mind Research Institute, Smoking Cessation Clinics, University of Sydney, Sydney, New South Wales, Australia

\begin{abstract}
Summary: Managing smoking cessation during pregnancy is vital to the wellbeing of the fetus and the mother. Women who continue to smoke during pregnancy expose the fetus to thousands of chemicals which have been shown to cause deleterious shortand long-term effects. Although a large majority of women cease smoking early in the pregnancy, many of them relapse following delivery. Following a review of current research, an overview of the safety and efficacy of smoking cessation treatments for pregnant women will be considered. Limited research has been performed in this field; however, it can be concluded that low-dose intermittent nicotine replacement therapy is a safe treatment modality for women who smoke during pregnancy. At present there has been no research on other current smoking cessation treatments; however, we will suggest techniques to improve cessation rates and strategies to reduce relapse.
\end{abstract}

Keywords: adolescence, complications, drugs (abuse), high-risk pregnancy

\section{INTRODUCTION}

Smoking is one of the most important modifiable causes of adverse outcomes during pregnancy associated with severe fetal and maternal morbidity and mortality. It is estimated that $22 \%$ of women smoke during pregnancy, but unfortunately only a few safe and effective smoking cessation treatments currently exist for pregnant women. ${ }^{1}$ Long-term research is lacking and controversy still exists as to the safety and efficacy of the current interventions, leaving many women with insufficient information regarding smoking cessation treatment. This review will highlight the current research and make recommendations on appropriate advice for smoking cessation treatments and recommendations for safe and effective treatment modalities for pregnant women.

\section{MEDICAL CONSEQUENCES OF SMOKING DURING PREGNANCY}

The adverse effects of smoking on the pregnancy can be seen throughout gestation. During the first trimester, smoking has been shown to increase the risk of miscarriage by $20-80 \%$ compared with non-smoking women. ${ }^{2}$ This increase in risk has been associated with a reduction in the levels of chorionic gonadotropin in women who smoke. Women who continue to smoke into the second trimester are at greater risk of developing placental abruption and placenta praevia compared with the general population due to carbon monoxide-induced hypoxia and placental enlargement, which causes the placenta to reach the cervical opening. ${ }^{3}$ The incidence of preterm deliveries and the incidence of very early preterm deliveries have also been shown to be greater in women who smoke during pregnancy. 4

Correspondence to: Assistant Professor Renee Bittoun

Email: bittounr@med.usyd.edu.au
For the fetus, cigarette smoking during pregnancy has been linked to many serious and preventable medical conditions; infants born to women who smoke during pregnancy are on average $200 \mathrm{~g}$ lighter. ${ }^{4}$ These infants also have a higher incidence of neonatal death and are at a greater risk of developing respiratory infections, asthma, atopy and otitis media. ${ }^{5}$ The research has found that women who quit smoking during the first trimester are at no greater risk of having a baby with low birth weight compared with non-smoking women.

The effects of smoking on neurological development have not been entirely defined; however, it has been documented that children born to women who smoke during pregnancy have cognitive, behavioural and developmental deficits:

- Cognitive impairment; ${ }^{6}$

- Impaired attention; ${ }^{7}$

- Poor impulse control; ${ }^{8}$ and

- Learning deficits. ${ }^{9}$

The effect of nicotine exposure via breast milk on infant morbidity is the one area of continual debate. The debate arises from the difficulty in separating the effects of prenatal exposure to postnatal exposure. Due to the lack of evidence, the American Academy of Pediatrics Committee on Drugs has removed nicotine from the list of drugs that are contraindicated in breast feeding. There are important differences between breast milk nicotine and plasma. Firstly, the half-life of nicotine in breast milk was measured to be about 95 minutes compared with 80 minutes in plasma. ${ }^{10}$ When a person smokes a cigarette, between $29 \%$ and $93 \%$ of the nicotine is absorbed, but there have been no reports describing how infants absorb nicotine from the gastrointestinal tract or the first-line metabolism of nicotine in infants. ${ }^{11}$ The mean intake of nicotine from breast milk has been shown to be approximately $7 \mu \mathrm{g} / \mathrm{kg} /$ day; it is currently not known whether these levels are deleterious to infant maturation, but studies in animals have shown that 
$2-6 \mathrm{mg} / \mathrm{kg} /$ day of nicotine can cause serious cell injury and damage. $^{12}$

Smoking has been shown to reduce daily milk production by approximately 250-300 g. ${ }^{13}$ One theory suggests that smoking induces an increase in hypothalamic dopamine, which in turn inhibits the production of prolactin. ${ }^{12,14}$ Women who smoke are also less likely to continue breast feeding beyond four months as one study showed the prevalence of breast feeding among smokers decreased from $63 \%$ to $43 \%$ compared with a decline of only $2 \%$ among non-smokers (90-88\%). The prevalence and duration of breast feeding has been linked to the daily number of cigarettes, with women who smoke more cigarettes less likely to continue breast feeding. ${ }^{15}$ In non-smokers, breast feeding has been conclusively shown to be protective against Sudden Infant Death Syndrome (SIDS), but this effect has not been shown among smokers. It is believed that exposure to nicotine from breast milk impairs infant arousal processes which may increase the risk of SIDS. ${ }^{16}$

\section{SMOKING CESSATION TREATMENTS}

Education is the first obstacle in reducing the number of pregnant women who smoke. Two recent studies looked at how many women received smoking cessation advice during their pregnancy. One study interviewed 274 general practitioners and 184 midwives and found that only about half provided advice to their patients. ${ }^{17}$ Another study interviewed 296 pregnant smokers to determine how often obstetric providers recommended smoking cessation medications and found that only $29 \%$ of respondents had a discussion about medications with their practitioner. ${ }^{18}$ All pregnant smokers should be given advice regarding smoking cessation. A meta-analysis reported that women who received smoking cessation intervention had a $70 \%$ improvement in cessation rates. ${ }^{19}$ The interventions consisted of one or more brief counselling sessions and self-help materials. Although abstinence early in pregnancy will produce the greatest benefits, clinicians should offer effective smoking cessation interventions throughout the course of pregnancy. A practitioner's advice about smoking cessation is therefore critical and should be tailored to pregnant women and offered to all pregnant smokers.

The lack of knowledge and intervention is demonstrated by the low number of women who stop smoking during pregnancy. In a study performed in Sweden, it was found that only $29 \%$ of pregnant smokers stopped smoking completely during pregnancy. The majority of these women (25\%) quit prior to or shortly after their first prenatal consultation. ${ }^{20}$ Thus, pregnant smokers who continue to smoke after their first prenatal visit are more likely to continue to smoke throughout the entire pregnancy. These women may require more intense intervention in order to quit.

Smoking cessation trials have revealed a reduction in low birth weight, a reduction in preterm birth and an increase in mean birth weight of $53 \mathrm{~g}$ for those in the intervention arm. ${ }^{4}$ Evidence suggests that psychosocial intervention including cognitive behavioural therapy (CBT), educational and motivational intervention strategies including feedback are the most effective in promoting efforts among pregnant smokers to quit. A meta-analysis performed by the United States Department of Health and Human Services found that abstinence rates were higher in the intervention group (16.8\%) compared with those who received no counselling or minimal care
(6.6\%). Interventions included advice about risks, pregnancyspecific self-help materials and follow-up. ${ }^{21}$ A second metaanalysis found that CBT and practitioner feedback had the highest efficacy with regards to abstinence, but it is expensive and therefore difficult to provide to all pregnant women. ${ }^{4}$

Most of the studies looking at pharmacotherapy have been limited to nicotine replacement therapy (NRT). The American College of Obstetrics and Gynecologists advocate that NRT products and other pharmacological agents should only be used when non-pharmacological treatments have failed. Although many studies have found improvement in abstinence rates in those treated with NRT, a recent meta-analysis found no significant advantage over other types of smoking cessation interventions. ${ }^{4}$ Some studies have shown that NRT is associated with an increased risk of congenital abnormalities, with one study suspending enrolment due to an increase in adverse events; the study found that the group treated with NRT (choice of patch, gum or lozenge) had a higher incidence of preeclampsia, preterm birth, placental abnormalities and fetuses small for gestational age. ${ }^{22}$ The study found a $60 \%$ increased risk (relative risk [RR] 1.61) of fetal abnormalities. The risk is even greater for musculoskeletal abnormalities (RR 2.63). The risk of abnormalities appears to be greater for those treated with patches as compared with those treated with gum and it is therefore recommended that pregnant women who smoke use intermittent rather than continuous nicotine replacement preparations and use the lowest dose possible to achieve control. ${ }^{23}$ It should also be recommended when possible to delay therapy until the second trimester in order to avoid the period of embryogenesis.

The antidepressant bupropion that has been specifically used to aid in smoking cessation has been found to increase abstinence rates among non-pregnant individuals compared with placebo. ${ }^{24}$ The mechanism for smoking cessation is currently not known. A recent cohort trial among 22 pregnant women receiving bupropion found that $10(45 \%)$ ceased smoking compared with three (14\%) of 22 controls. ${ }^{25}$ Another study suggests that bupropion may be safe to use in pregnancy with no increased risk of teratogenic effects when used during the first trimester compared with other antidepressants or when used outside of the first trimester. ${ }^{26}$ More research is necessary in order to make recommendations for the use of bupropion during pregnancy.

The most recent smoking cessation treatment varenicline that agonizes and blocks alpha- 4 beta- 2 nicotinic acetylcholine receptors has been extensively studied in the general population, but no study has yet been completed among pregnant women.

The effectiveness of alternative therapies including acupuncture, acupressure, laser therapy or electrostimulation has been extensively studied for smoking cessation. A recent meta-analysis identified 22 studies that compared a form of acupuncture and related therapies with a sham therapy and found no evidence that these therapies are effective for smoking cessation. ${ }^{27}$ It must be noted that the review did not look at the effectiveness of alternative therapies in pregnancy.

\section{POSTPARTUM RELAPSE PREVENTION}

Of the large number of women who successfully quit smoking during pregnancy, many women relapse after delivery. The ranges of relapse rates are broad because of the variety of cessation intervention methods. Studies have shown that between 
$29 \%$ and $85 \%$ of women who received an intervention plan relapsed after delivery. ${ }^{28}$ The risks for relapsing were commonly reported as having a partner or household member who smokes, pre- and postpartum depression and the pressure of taking care of a new baby. ${ }^{29,30}$

From the current research it is clear that smoking during pregnancy may lead to adverse complications for both the mother and the fetus. Research has shown that smoking cessation interventions can reverse many of the adverse effects caused by smoking; however, many obstacles prevent widespread implementation of smoking cessation interventions in pregnancy in clinical practice. Many of the obstacles described revolve around communication difficulties between staff members, staff attitudes and perceptions about interventions, perceived lack of skills and training and lack of quality programmes that are acceptable to women.

\section{EVIDENCE-BASED BEHAVIOURAL THERAPIES}

It is suggested that practitioners screen all pregnant women for smoking using developed guidelines based on the " 5 A's":

- Asking all pregnant women if they smoke;

- Advising all pregnant women of the risks associated with smoking during pregnancy and benefits of quitting;

- Assisting all pregnant women to quit;

- Assessing the women's readiness to change and;

- Asking and assisting at every opportunity.

\section{SOME EVIDENCE-BASED BEHAVIOURAL TIPS}

The advice listed below can be implemented by any woman who smokes and have been shown to help avoid relapse:

- Everyone smokes outside; ${ }^{31}$

- Everyone smokes outside the car; ${ }^{32}$

- Reduce caffeine intake; ${ }^{33}$

- Reduce alcohol intake; ${ }^{32}$

- Exercise during acute urges; ${ }^{34}$

- Avoid quitting during times of stress or major change; ${ }^{35}$

- Quitting with a buddy has not conclusively been shown to increase abstinence rates; ${ }^{36}$ and

- Avoiding triggers or cues such as alcohol may not help. ${ }^{37}$

\section{CONCLUSION}

The importance of screening all pregnant women and providing them with information about smoking during pregnancy can be seen in the large proportion of women who quit before or shortly after the first prenatal visit. On account of the serious risks to the fetus and the pregnant woman every woman who smokes should be offered pregnancy-tailored psychosocial smoking cessation counselling throughout pregnancy. CBT is the most effective smoking cessation treatment for pregnant women, but owing to extensive cost and time, CBT cannot be offered to every patient and therefore cannot be implemented on a wide-scale setting. For those women who find it difficult to quit or for those with moderate to high levels of addiction, NRT should be offered to any patient for whom the therapy is not contraindicated as the potential benefits of quitting outweigh the risk of the pharmacotherapy. It is the opinion of the authors that NRT should be started at the lowest possible dose and should be used intermittently rather than continuously under the supervision of a physician. The most effective strategy to achieve abstinence would be to combine CBT with counselling and NRT.

Disclosure: Renee Bittoun has received unencumbered funds from the manufacturers of smoking cessation products.

\section{REFERENCES}

1 Laws PJ, Sullivan EA. Report on the evaluation of the Perinatal National Minimum Data Set 2004. Australian Institute of Health and Welfare. Cat. No. PER 27. Sydney. AIHW National Perinatal Statistics Unit (Perinatal Statistics Series No. 14)

2 Castles A, Adams EK, Melvin CL, Kelsch C, Boulton ML. Effects of smoking during pregnancy. Five meta-analyses. Am J Prev Med 1999;16:208-15

3 Cnattingius S. The epidemiology of smoking during pregnancy: smoking prevalence, maternal characteristics, and pregnancy outcomes. Nicotine Tob Res 2004;6(Suppl. 2):S125-40

4 Jauniaux E, Burton GJ. Morphological and biological effects of maternal exposure to tobacco smoke on the fetoplacental unit. Early Hum Dev 2007;3:699-706

5 Shea AK, Steiner M. Cigarette smoking during pregnancy. Nicotine Tob Res 2008;10:267-78

6 Butler NR, Goldstein H. Smoking in pregnancy and subsequent child development. Br Med J 1973;4:573-5

7 Picone TA, Allen LH, Olsen PN, Ferris ME. Pregnancy outcome in North American women. II. Effects of diet, smoking cessation and weight gain on placentas and on neonatal physical and behavioural characteristics. Am J Clin Nutr 1982;36:1214-24

8 Kristjansson EA, Fried PA, Watkinson B. Maternal smoking during pregnancy affects children's vigilance performance. Drug Alcohol Depend 1989:11-19

9 Fried PA. Cigarettes and marijuana: are there measurable long-term neurobehavioral teratogenic effects? Neurotoxicology 1989;10:577-83

10 Dorea JG. Maternal smoking and infant feeding: breastfeeding is better and safer. Mater Child Health J 2007;11:287-91

11 Dahlstrom A, Ebersjo C, Lundell B. Nicotine exposure in breastfed infants. Acta Paediatr 2004;93:810-6

12 Anderson AN, Lund-Andersen C, Larsen JF, et al. Suppressed prolactin but normal neurophysin levels in cigarette smoking breast-feeding women. Clin Endocrinol 1982;17:363-8

13 Hopkinson JM, Schanler RJ, Fraley JK, Garza C. Milk production by mothers of premature infants: influence of cigarette smoking. Pediatrics 1992;90:934-8

14 Widstrom AM, Werner S, Matthiesen AS, Svensson K, Uvnäs-Moberg K. Somatostatin levels in plasma in non-smoking and smoking breastfeeding women. Acta Paediatr Scand 1991;80:13-21

15 Najdawi F, Faouri M. Maternal smoking and breast feeding. Eastern Mediterranean Health J 1999;5:450-6

16 Richardson HL, Walker AM, Horne RSC. Maternal smoking impairs arousal patterns in sleeping infants. Sleep 2009;32:515-21

17 Pullon S, Webster M. Smoking cessation and nicotine replacement therapy in current primary maternity care. Austral Fam Phys 2004;33:94-6

18 Rigotti NA, Park ER, Chang Y, Regan S. Smoking cessation medication use among pregnant and postpartum women. Obstet Gynecol 2008;111:348-55

19 Lumley J, Chamberlain C, Dowswell T, Oliver S, Oakley L, Watson L. Interventions for promoting smoking cessation during pregnancy. Cochrane Database Systemic Rev 2009, Issue 3. Art. No.: CD001055. DOI:10.1002/ 14651858. CD001055.pub.3;1-163

20 Cnattingius S. The epidemiology of smoking during pregnancy: smoking prevalence, maternal characteristics, and pregnancy outcomes. Nicotine Tob Res 2004;6(Suppl. 2):S125-40

21 Melvin CL, Dolan-Mullen P, Windsor RA, Whiteside HP Jr, Goldenberg RL. Recommended cessation counselling for pregnant women who smoke: a review of the evidence. Tobacco Control 2000:9(suppl 3);iii80-iii84

22 Pollack KI, Oncken CA, Lipkus IM, Lyna P, Swamy GK. Nicotine replacement and behavioral therapy for smoking cessation in pregnancy. Am J Prev Med 2007;33:297-305

23 Dempsey DA, Benowitz NL. Risks and benefits of nicotine to aid smoking cessation in pregnancy. Drug Saf 2001;24:277-322

24 Jorenby DE, Leischow SJ, Nides MA, et al. A controlled trial of sustained release buproprion, a nicotine patch or both for smoking cessation. $N$ Engl J Med 1999;340:685-91 
25 Chan B, Einarson A, Koren G. Effectiveness of bupropion for smoking cessation in pregnancy. J Addict Dis 2005;24:19-23

26 Cole JA, Modell JG, Haight BR, Cosmastos IR. Bupropion in pregnancy and the prevalence of congenital malformations. Pharmacoepidemiol Drug Saf 2007;16:474-84

27 White AR, Rampes H, Ernst E. Acupuncture for smoking cessation. Cochrane Database Systemic Review 2006, Issue 1. Art. No.: CD000009.DOI:10.1002/ 14651858. CD000009.pub2.;1-43

28 Fang WL, Goldstein AO, Butzen AY, et al. Smoking cessation in pregnancy: a review of postpartum relapse prevention strategies. J Am Board Fam Pract/Am Board Fam Pract 2004;17:264-75

29 Merzel C, English K, Moon-Howard J. Identifying women at-risk for smoking resumption after pregnancy. Maternal and Child Health Journal 2009; DOI.10.1007/S10995-009-0502-1

30 Hymowitz N, Schwab M, McNerney C, Schwab J, Eckholdt H, Haddock K. Postpartum relapse to cigarette smoking in inner city women. J Natl Med Assoc 2003;95:461-74

31 Gilpin EA, White MM, Farkas AJ, Pierce J. Home smoking restrictions: which smokers have them and how they are associated with smoking behaviour. Nicotine Tob Res 1999;1:153-62
32 Garvey AJ, Bliss RE, Hitchcock JL. Predictors of smoking relapse amongst self-quitters: a report of the normative aging study. Addict Behav 1992;17:367-77

33 Swanson JA, Lee JW, Hopp J. Caffeine and nicotine: a review of their joint use and possible interactive effects in tobacco withdrawal. Addict Behav 1994;19:229-56

34 Taylor AH, Ussher M, Faulkner G. The acute effects of exercise on cigarette cravings, withdrawal symptoms, affect and smoking behaviour: a systematic review. Addiction 2007;102:534-43

35 O'Hara P, Portser SA, Anderson BP. The influence of menstrual cycle changes on the tobacco withdrawal syndrome in women. Addict Behav 1989;14:595-600

36 May S, West R, Hajek P, McEwen A, McRobbie H. Randomised controlled trial of social support ('buddy') intervention for smoking cessation. Patient Educ Counsel 2006;64:235-41

37 Thewissen R, van der Meijden VA, Havermans R. From the office to the pub: the role of smoking - relevant contexts and cue-elicited urge to smoke. Eur Addict Res 2008;14:198-205 